\title{
Early QTe Interval Prolongation After Primary Percutaneous Coronary Intervention May Have a Positive Impact
}

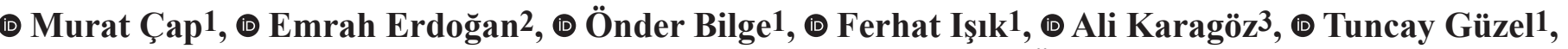

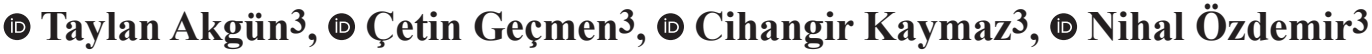

\footnotetext{
${ }^{1}$ University of Health Sciences Turkey, Gazi Yaşargil Training and Research Hospital, Department of Cardiology, Diyarbakır, Turkey ${ }^{2}$ Yüzüncü Y1l University Faculty of Medicine, Department of Cardiology, Van, Turkey

${ }^{3}$ University of Health Sciences Turkey, Kartal Koşuyolu Training and Research Hospital, Department of Cardiology, İstanbul, Turkey
}

\begin{abstract}
Objectives: Corrected QT (QTc) interval is prolonged in acute myocardial infarction and begins to shorten after successful reperfusion. Data on the early change of QTc after reperfusion and the prognostic significance of this change are limited. We aimed to evaluate the change of QTc interval in the first hour following successful primary percutaneous coronary intervention (pPCI) in ST-elevation myocardial infarction (STEMI) patients and its relationship with reperfusion parameters such as myocardial blush grade (MBG) and ST-segment resolution (STR\%).

Materials and Methods: Patients who presented with the first STEMI episode and underwent successful pPCI were included in the study. After pPCI, MBG and STR\% were calculated. QTc measurements were made from the electrocardiography (ECG) recorded at admission (Pre-pPCI QTc), 1 hour after pPCI (Post-pPCI QTc), and the 24 $4^{\text {th }}$ hour.

Results: One hundred and five patients who had successful pPCI with adequate ECG data were enrolled in the study. The mean Pre-pPCI QTc was $409 \pm 34 \mathrm{~ms}$, and the mean post-pPCI QTc was $427 \pm 32 \mathrm{~ms}$. A statistically significant prolongation was observed in the QTc interval after pPCI (QTc-Change) [21 ms (-3, 37 interquartile range (IQR)), $\mathrm{p}<0.001]$. The median STR was 71\% (60-83 IQR), and the median MBG was 2 (1-3 IQR). In the multivariable linear regression analysis, a significant relationship was observed between QTc-Change with MBG and STR $\%[\beta=9.077,95 \%$ confidence interval (CI): $2.55-15.60, p=0.006$ and $\beta=9.315,95 \% \mathrm{CI}: 2.00-16.62, \mathrm{p}=0.013$, respectively].
\end{abstract}

Address for Correspondence: Murat Çap, University of Health Sciences Turkey, Gazi Yaşargil Training and Research Hospital,

Department of Cardiology, Diyarbakır, Turkey

e-mail: murat00418@hotmail.com ORCID: orcid.org/0000-0003-1164-2124

Received: 12.02.2021 Accepted: 18.05.2021

Cite this article as: Çap M, Erdoğan E, Bilge Ö, Işık F, Karagöz A, Güzel T, Akgün T, Geçmen Ç, Kaymaz C, Özdemir N. Early

QTc Interval Prolongation After Primary Percutaneous Coronary Intervention May Have a Positive Impact. EJCM 2021;9(2):105-112.

DOI: 10.32596/ejcm.galenos.2021-02-013

${ }^{\circ}$ Copyright 2021 by Heart and Health Foundation of Turkey (TÜSAV) / E Journal of Cardiovascular Medicine published by Galenos Publishing House. 
Conclusion: It was found that the QTc interval continued to prolong somewhat in the early period after successful pPCI in STEMI patients, and this QTc-Change was significantly associated with reperfusion parameters such as MBG and STR\%. STR\% and MBG were higher in those with higher QTc-Change.

Keywords: ST-segment elevation myocardial infarction, QTc interval, myocardial blush grade, ST-segment resolution

\section{Introduction}

The QT interval on the surface electrocardiogram (ECG) represents the time from the onset of ventricular depolarization to the completion of repolarization. Previous studies have shown that prolonged corrected QT (QTc) is associated with high arrhythmic event rates, sudden death, and all-cause mortality in the normal population and patients with acute myocardial infarction $(\mathrm{AMI})^{(1-5)}$.

Acute myocardial ischemia has been shown to increase repolarization heterogeneity and prolong the QT interval ${ }^{(5,6)}$. Studies have shown that the QTc interval is prolonged in AMI and begins to shorten after successful reperfusion ${ }^{(7-9)}$. However, data on the change of the QTc interval in the early period after revascularization and the prognostic significance of this change are limited. In a study by Bonnemeier et al. ${ }^{(7)}$, it was found that in patients with AMI, the OTc interval initially became prolonged in the first hour following successful primary percutaneous coronary intervention (pPCI) but it was then followed by OTc interval shortening.

ST-segment resolution (STR\%) and myocardial blush grade (MBG) are among the early reperfusion parameters that have shown prognostic significance in ST-elevation myocardial infarction (STEMI) patients ${ }^{(10-13)}$. Our study aimed to evaluate the change of QTc interval during the following hour after successful pPCI in STEMI patients and its relationship to reperfusion parameters such as MBG and STR\%.

\section{Materials and Methods}

A total of 150 consecutive patients admitted with STEMI were evaluated. The patients enrolled were initially selected from those with clinical history and symptoms suggestive of the first episode of acute STEMI, who had presented within 12 hours after the onset of symptoms. According to current guidelines, the diagnosis STEMI required: Chest pain lasting $>20 \mathrm{~min}$ and STelevation (STE) of $>1 \mathrm{~mm}$ in at last two contiguous ECG leads without left ventricular hypertrophy and left bundle branch block ${ }^{(14)}$. Only patients who underwent successful pPCI with thrombolysis in myocardial infarction (TIMI) flow grade 3 were included. After exclusion criteria were applied, 105 patients were enrolled in the study.

Patients excluded from the study were those with non-sinus rhythm, bundle branch block, pacemaker rhythm, prior AMI or coronary bypass grafting, coronary occlusions unsuitable for PCI, TIMI flow grade $<3$, and challenge to determine the end of T-waves.

\section{Angiographic Examination and Definition}

Coronary angiography, pPCI, and periprocedural care conformed to the current guidelines ${ }^{(14)}$. The culprit lesion was crossed with wire within the first 60 minutes of hospital admission in all the cases. After successful revascularization (TIMI-3 flow), MBG was calculated. TIMI flow grade has been defined as follows: TIMI 0 refers to the absence of any antegrade flow beyond a coronary occlusion; TIMI 1 flow is faint antegrade coronary flow beyond the occlusion, although filling of the distal coronary bed is incomplete; TIMI 2 flow is delayed or sluggish antegrade flow with complete filling of the distal territory; TIMI 3 flow is normal flow, which fills the distal coronary bed completely ${ }^{(15)}$. MBG is an angiographic measure of myocardial perfusion. MBG has been defined as follows: 0, no myocardial blush (MB) or 
contrast density (CD); 1, minimal MB or CD; 2, moderate $\mathrm{MB}$ or $\mathrm{CD}$ but less than that obtained during angiography of a non-infarct related coronary artery; 3 , standard MB or $\mathrm{CD}$, comparable with that obtained during angiography of a non-infarct-related coronary artery ${ }^{(13)}$.

\section{Electrocardiographic Analysis}

For each patient, standard 12-lead-ECGs (paper speed of $25 \mathrm{~mm} / \mathrm{s}$, standardization of $10 \mathrm{~mm} / 1 \mathrm{mV}$ ) were recorded at admission, within the first hour after pPCI, and at the 24 hours after pPCI. All analyses were made by two independent observers using a magnifying glass. ECG was performed with the Nihon Kohden Electrocardiograph (model ECG 2350).

ST-Segment Resolution (STR\%): STR was calculated from the ECGs taken at admission and the first hour after pPCI. The sum of STR was measured 20 milliseconds after the end of QRS complex: Leads V1-6 for anterior MI and leads II, III, and aVF for inferior infarction. ST resolution was calculated as a percentage reduction of the absolute STE in the single lead, which is associated with the infarct territory with maximum STE on the baseline $\mathrm{ECG}^{(10)}$.

QTe Interval Measurement: QT intervals were measured from the recorded ECGs (baseline, $1^{\text {st }}$ and $24^{\text {th }}$ hours after $\left.\mathrm{pPCI}\right)$. Measurements were made using a manual compass in either lead II for inferior MI and V2 or V5 for anterior MI, with the most prolonged QT interval being used. The maximum measured interval in successive 3-5 beats was taken. When leads II, V2, or V5 were deemed unsuitable, one of the remaining leads associated with infarct territory was chosen. QT intervals were measured from the onset of the QRS complex to the point of return of the $\mathrm{T}$ wave to the isoelectric line or the nadir between the $T$ and $U$ waves in cases the $U$ wave was present. QT intervals were corrected for heart rate effects using a modified Bazzet's formula $(\mathrm{QTc}=\mathrm{QT} /(\mathrm{R}-\mathrm{R}) 1 / 2)$.

Transthoracic echocardiography was performed 24 hours after admission, and the left ventricular ejection fraction (LVEF \%) was measured by the modified Simpson method.
The study approved by the local institutional ethics committee. The study protocol conforms to the Declaration of Helsinki [University of Health Sciences Turkey, Gazi Yaşargil Training and Research Hospital Ethics Committee (approval date: 28/02/2020 number: 413].

\section{Statistical Analysis}

Statistical analysis was performed by using R software version 4.02 ( $\mathrm{R}$ Project, Austria Vienna). Continuous variables were presented as mean \pm standard deviations, or if a non-normal distribution was found, they were given as median, interquartile range: $25-75 \%$ (IQR). Categorical variables were expressed as percentages. The histogram and Shapiro-Wilks test were used to verify the normal distribution of data. The paired Student's t-test was used to assess QTc change after pPCI. Correlation between variables was performed using Spearman's rank or Pearson correlation test according to the distribution of data.

Outcome Variable: QTc-Change first hour after pPCI.

Statistical Modeling: Multiple linear regression analysis was used to assess the relationship of QTcChange with the STR\% and MBG after adjusting common clinical predictors (age, gender, diabetes mellitus, hypertension, peak troponin, infarct location, symptom to door time, potassium, Glycoprotein IIb/IIIa inhibitor use). Continuous variables were included in the model by restricted cubic spline transformation ( 3 knot) to attain the nonlinearity of data. Continuous variables were presented in regression analysis as their interquartile range (25-75 IQR). In all the statistical analyses, a p-value of $<0.05$ was considered statistically significant. The correction for p-value was performed where needed.

\section{Results}

One hundred and five patients who had successful pPCI with adequate ECG data were enrolled in the study. The mean age was $55 \pm 10$ years in the study population. Nineteen (18\%) patients were female, and 44 (42\%) patients had anterior STEMI. Baseline characteristics, 
clinical, laboratory, and electrocardiographic findings of patients were given in Table 1.

The mean Pre-pPCI QTc was $409 \pm 34 \mathrm{~ms}$, the mean post-pPCI QTc was $427 \pm 32 \mathrm{~ms}$, and the mean $24^{\text {th }}$ hour QTc was $424 \pm 34 \mathrm{~ms}$ (Figure 1). After pPCI, the median $21 \mathrm{~ms}(-3,37 \mathrm{IQR})$ prolongation was observed in the QTc interval and this prolongation (QTc-Change) was statistically significant $(\mathrm{p}<0.001)$. The median STR was $71 \%$ (60-83 IQR), the median MBG was 2 (1-3 IQR), the median symptom to door time was 3 (1.75-4 IQR) hours. The median LVEF measured at 24 hours was 55\% (4757 IQR). In-hospital mortality occurred in one patient who presented with anterior STEMI, and non-sustained ventricular tachycardia was observed within the first 24 hours after revascularization in another patient.

A weak negative correlation was observed between admission Pre-pPCI QTc and STR (\%) ( $\mathrm{r}=-0.237)$, LVEF (\%) $(\mathrm{r}=-0.383)$ and $\mathrm{MBG}(\mathrm{r}=-0.355)$. A weak positive correlation was observed between symptom to door time and pre-pPCI QTc $(r=0.382)$. A moderate negative correlation was observed between QTc-Change and PrepPCI QTc $(\mathrm{r}=-0.422)$. A moderate positive correlation was observed between QTc change and STR\% $(r=0.402)$, MBG ( $\mathrm{r}=407)$, and LVEF\% $(\mathrm{r}=0.437)$. A weak negative correlation was observed between QTc-Change and symptom to door time $(\mathrm{r}=-333)$. In the multivariable linear regression analysis, a significant relationship was

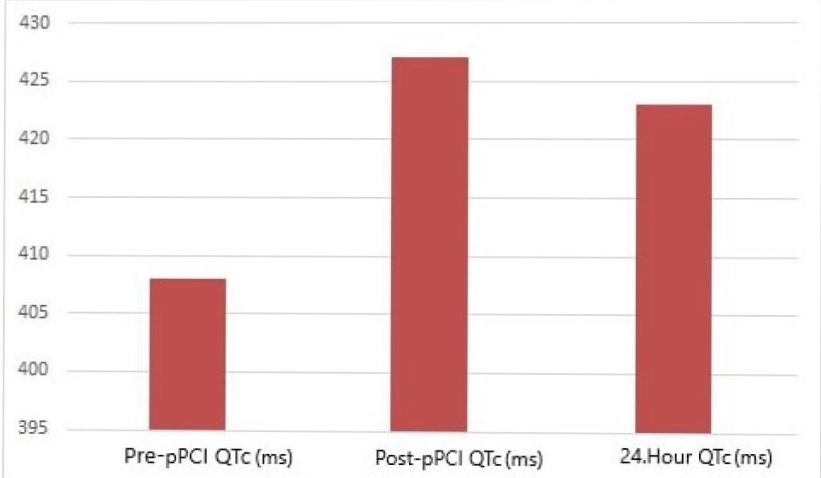

Figure 1. Temporal change of QTc interval: Pre-pPCI QTc, PostpPCI QTc, and $24^{\text {th }}$-hour QTc

pPCI: Primary percutaneous coronary intervention, QTc: Corrected QT
Table 1. Baseline characteristics, and clinical, laboratory, and electrocardiographic findings of patients

\begin{tabular}{|c|c|}
\hline & Total $(n=105)$ \\
\hline Age (year) & $55 \pm 10$ \\
\hline Gender (female) & $19(18 \%)$ \\
\hline Diabetes mellitus & $14(13 \%)$ \\
\hline Hypertension & $35(33 \%)$ \\
\hline Smoking & $34(32 \%)$ \\
\hline Hyperlipidemia & $36(34 \%)$ \\
\hline Symptom to door time (h) & $3(1.75-4)$ \\
\hline Troponin (ng/mL) & $3.8(0.34-21)$ \\
\hline Peak troponin (ng/mL) & $34(13-50)$ \\
\hline Killip class $>1$ & $21(20 \%)$ \\
\hline Infarct location (anterior) & $44(42 \%)$ \\
\hline \multicolumn{2}{|l|}{ Infarct vessel } \\
\hline Left anterior descending & $44(42 \%)$ \\
\hline Left circumflex & $23(22 \%)$ \\
\hline Right & $38(36 \%)$ \\
\hline Left ventricular ejection fraction (\%) & $55 \%(47-57)$ \\
\hline ST-segment resolution (\%) & $71(60-83)$ \\
\hline \multicolumn{2}{|l|}{ Myocardial blush grade } \\
\hline 0 & $6(6 \%)$ \\
\hline 1 & $20(19 \%)$ \\
\hline 2 & $44(42 \%)$ \\
\hline 3 & $35(33 \%)$ \\
\hline Glycoprotein Ilb/Illa inhibitor use & $40(38 \%)$ \\
\hline Hemoglobin (g/dL) & $13.7 \pm 1.7$ \\
\hline Creatinine (mg/dL) & $0.80 \pm 0.18$ \\
\hline Potassium (mmol/L) & $4.03 \pm 0.35$ \\
\hline Heart rate pre-pPCI (beat/min) & $75 \pm 17$ \\
\hline Heart rate post-pPCI (beat/min) & $76 \pm 14$ \\
\hline Heart rate $24^{\text {th }}$ hour (beat/min) & $71 \pm 11$ \\
\hline Pre-pPCI QT interval (ms) & $371 \pm 39$ \\
\hline Post-pPCI QT interval (ms) & $381 \pm 36$ \\
\hline $24^{\text {th }}$ hour QT interval (ms) & $392 \pm 37$ \\
\hline Pre-pPCI QTc interval (ms) & $409 \pm 34$ \\
\hline Post-pPCI QTc interval (ms) & $427 \pm 32$ \\
\hline $24^{\text {th }}$-hour QTc interval (ms) & $424 \pm 34$ \\
\hline QTC-Change (ms) & $21(-3,37)$ \\
\hline
\end{tabular}


observed between QTc-Change with MBG and STR\% ( $\beta$-coefficient $=9.077, \quad 95 \% \quad \mathrm{CI}: \quad 2.55-15.60, \quad \mathrm{p}=0.006$ and $\beta$ coefficient $=9.315,95 \%$ CI: $2.00-16.62, p=0.013$, respectively). Multivariable linear regression analysis between the QTc-Change and clinical variable was given in Table 2. Partial effect plots of MBG and STR\% with QTc-Change were given in Figure $2 \mathrm{a}$ and $2 \mathrm{~b}$. It is seen that as STR\% and MBG increase, QTc-Change increases. In Figure 3, we summarized the relative importance of each predictor in the model. MBG was ranked as the most contributing predictor for QTc-Change, and followed by STR $\%$.

\section{Discussion}

There are insufficient data regarding the prognostic significance of the change in the QTc interval after revascularization in STEMI patients, and our study is important in terms of showing the relationship between this QTc-change and prognostic parameters. This study showed that the QTc interval was significantly prolonged

Table 2. Multivariable Linear Regression analysis of QTc-Change with clinical variables

\begin{tabular}{|c|c|c|c|}
\hline & $\beta$-coefficient & $95 \% \mathrm{Cl}$ & p-value \\
\hline Age (year) (from 48 to 61 ) & 3.42 & $-4.62,8.98$ & 0.775 \\
\hline Sex (male/female) & 5.18 & $-8.79,19.06$ & 0.460 \\
\hline Hypertension (from 0 to 1 ) & 2.84 & $-9.12,14.81$ & 0.637 \\
\hline Diabetes mellitus (from 0 to 1 ) & -5.20 & $-20.98,10.56$ & 0.513 \\
\hline Troponin $(\mathrm{ng} / \mathrm{mL}$ ) (from 0.34 to 18.9 ) & -7.18 & $-11.33,17.12$ & 0.703 \\
\hline Symptom to door time (hour) (from 2 to 4 ) & -8.79 & $-8.79,6.37$ & 0.07 \\
\hline Infarct type (anterior/nonanterior) & 1.59 & $-9.29,12.48$ & 0.772 \\
\hline STR\% (from 60 to 83 ) & 9.31 & $2.00,16.62$ & 0.013 \\
\hline MBG (from 1 to 3 ) & 9.07 & $2.55,15.60$ & 0.006 \\
\hline Glycoprotein Ilb/IIla inhibitor & 4.81 & $-4.86,14.48$ & 0.320 \\
\hline Potassium (3.8 to 4.2$)$ (mmol/L) & -4.56 & $-11.21,2.09$ & 0.039 \\
\hline
\end{tabular}

Regression coefficients were given according to the IQR 25-75\% (interquartile-range) changes of the continuous variables. Because of nonlinearity of some data (we used restrictive cubic splines $3 \mathrm{knot}$ ) such-as Potassium, corresponding $\mathrm{Cl}$ includes zero; however, $p$-value is significant.

STR: ST-segment resolution, MBG: Myocardial blush grade, Cl: Confidence interval.

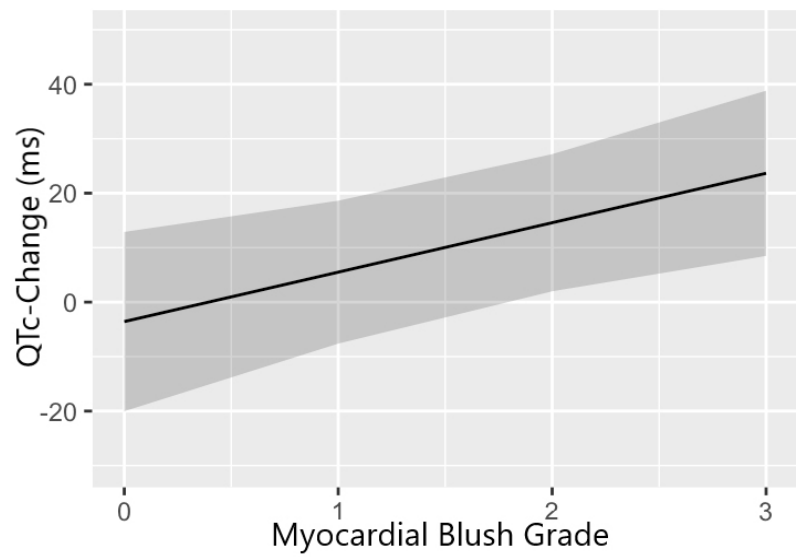

Figure 2a. Partial effect plot in the model for QTc-change and myocardial blush grade

QTc: Corrected QT

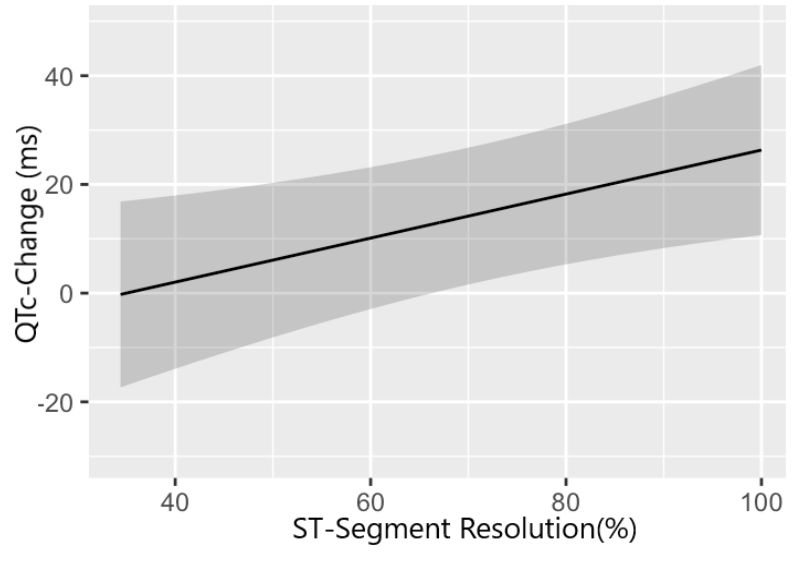

Figure 2b. Partial effect plot in the model for QTc-Change and ST-Segment resolution (\%)

QTc: Corrected QT 


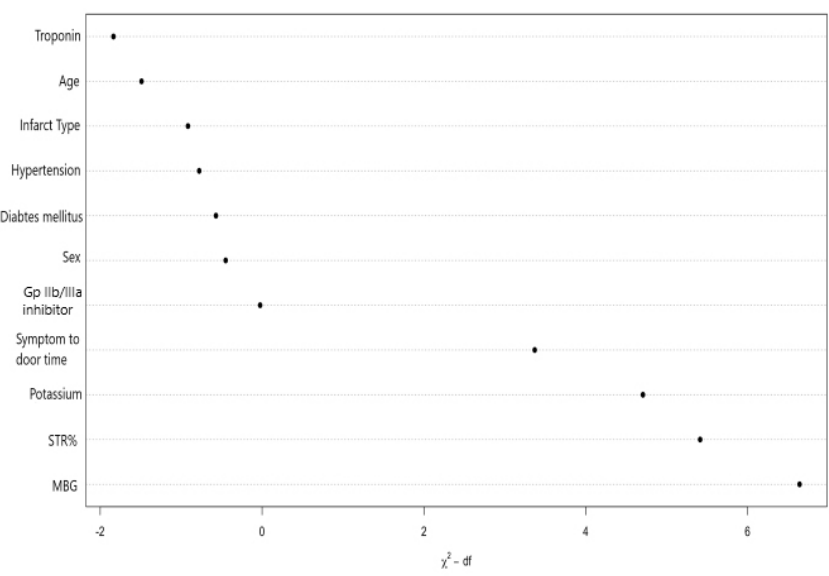

Figure 3. Relative importance of each variable in the model for QTC-change; STR\%: ST-segment resolution (\%), MBG: Myocardial blush grade

in the early period after successful reperfusion in STEMI patients. QTc-Change was significantly associated with STR\% and MBG by multivariable linear regression analysis, and $\mathrm{STR} \%$ and $\mathrm{MBG}$ were higher in patients with prolonged QTc interval. Moreover, the QTc change demonstrated a moderately negative correlation with both LVEF\% and Pre-pPCI QTc.

Acute myocardial ischemia has been shown to modify the QT interval duration, increase repolarization heterogeneity, and prolong the maximum electrocardiographic QT interval ${ }^{(6)}$. Numerous mechanisms have been proposed for ischemia to prolong the QTc duration. Increased sympathetic activity, together with structural myocardial damage, electrolyte imbalance, and dysfunction of the ion channels in the acute phase of ischemia, may cause QT prolongation ${ }^{(9)}$. Many studies have shown that a long admission QTc interval is associated with a poor prognosis in AMI. In one study, a QTc interval $>445 \mathrm{~ms}$ on admission to the emergency department was an independent predictor for all-cause mortality and heart failure in STEMI patients ${ }^{(16)}$. In our study, the Pre-pPCI QTc interval, measured on admission to the emergency department, showed a negative correlation with STR\%, MBG, and LVEF\%, respectively. Again, the Pre-pPCI
QTc interval was prolonged in patients with increased symptom to door time in our study.

Although many studies in patients with AMI report shortening of the QTc interval after successful reperfusion, studies on the QTc interval change during the following hour after reperfusion are limited. Reperfusion may affect the QTc interval, either directly by influencing the electrophysiological milieu or indirectly by interfering with cardiac autonomic nervous control ${ }^{(17)}$. In a study by Bonnemeier et al. ${ }^{(7)}$, it was found that the QTc interval continued to lengthen in the first hour after reperfusion in AMI patients who underwent pPCI and then began to shorten following hours. In our study, a significant prolongation was observed in the QTc interval (QTcChange) at the 1 st hour after successful pPCI $[21 \mathrm{~ms} \mathrm{(-3,}$ 37 IQR) p-value $<0.001$ ], and although a decrease was observed in the QTC interval measured at the $24^{\text {th }}$ hour, it was still found to be higher than the baseline. MBG is an angiographic measure of myocardial perfusion and a strong predictor of mortality in patients with TIMI-3 flow after $\mathrm{pPCI}^{(12)}$. Furthermore, STR\% is an electrocardiographic indicator of myocardial perfusion and has a strong relationship with mortality and reinfarction ${ }^{(10)}$. Even if TIMI-3 flow is provided in the infarct-related vessel, there may be a problem in myocardial perfusion at the microvascular level. Therefore, patients with low STR\% and $\mathrm{MBG}$ are associated with heart failure and poor outcomes $^{(10-12)}$. In the study of Poli et al. ${ }^{(13)}$, patients with both MBG $2 / 3$ and STR $>50 \%$ immediately after pPCI showed a significant 7-day and 6-month functional recovery on echocardiography. In our study, a significant relationship was observed between QTc-Change with $\mathrm{STR} \%$ and $\mathrm{MBG}$ in regression analysis. STR\%, MBG, and LVEF were higher in patients with prolongation of QTc interval after pPCI. QTc-Change was lower in patients with increased symptom to door time.

In one study evaluating patients who underwent elective PCI, it was observed that QTc was prolonged in $100 \%$ of patients during balloon inflation and began to decrease immediately after deflation, and QTc prolongation 
could be the earliest sign of transmural ischemia ${ }^{(18)}$. In a study of AMI patients comparing successful with unsuccessful reperfusion, it was found that the QTc interval peaked towards the $12^{\text {th }}$ hour in the revascularization group and started to shorten afterward, and it was observed that it continued to increase without peaking in the patient group without reperfusion. LVEF\% was better in the patient groups with transient prolongation ${ }^{(19)}$. In our study, LVEF\% measured at the $24^{\text {th }}$ hour was higher in patients with a prolonged QTc interval after reperfusion. Considering that cellular mechanisms are disrupted for a longer period in myocardial infarction than in elective balloon angioplasty (just as echocardiographic stunning does not improve immediately after reperfusion), the QT interval may continue to prolong for a while until the electrophysiological factors that cause QT prolongation recover and then begin to shorten. Reflecting on the relationship of QTc-Change with prognostic parameters such as STR, MBG, $\mathrm{LVEF} \%$, and symptom to door time, the moderate prolongation in the early period in the QTc interval may be an indicator of viable myocardial tissue, just like in myocardial stunning. The reversible impairment of ventricular repolarization after reperfusion for patients without adverse event may be interpreted as "electrical stunning" of the ventricular myocardium ${ }^{(7)}$.

The moderate prolongation of the QTc interval in the early hours after pPCI may be prognostically valuable due to its association with better myocardial perfusion and better left ventricular function in some patient groups.

\section{Study Limitations}

An important limitation of this study was the small cohort of patients. Another limitation of the study was that the QTc interval change was not monitored after the first hour. Perhaps, examining the relationship of QT prolongation with parameters such as the infarct area may be valuable in terms of showing the presence of living tissue.

\section{Conclusion}

It was found that the QTc interval continued to prolong somewhat in the early period after successful pPCI in STEMI patients, and this QTc-Change was significantly associated with reperfusion parameters such as MBG and STR\%. STR\% and MBG were higher in those with higher QTc-Change.

\section{Ethics}

Ethics Committee Approval: This study was approved by the University of Health Sciences Turkey, Gazi Yaşargil Training and Research Hospital Ethics Committee (approval date: 28/02/2020, number: 413).

Informed Consent: Informed consent form was obtained.

Peer-review: Internally and externally peer-reviewed.

\section{Authorship Contributions}

Surgical and Medical Practices: M.Ç., Concept: M.Ç., T.A., Ç.G., Design: M.Ç., Ö.B., F.I., A.K., Data Collection or Processing: M.Ç., Ö.B., F.I., T.G., Analysis or Interpretation: A.K., T.A., Ç.G., Literature Search: E.E., Ö.B., Writing: M.Ç., E.E., C.K., N.Ö.

Conflict of Interest: The authors report no conflicts of interest.

Financial Disclosure: The authors declare that this study has received no financial support.

\section{References}

1. Straus SM, Kors JA, De Bruin ML, et al. Prolonged QTc interval and risk of sudden cardiac death in a population of older adults. J Am Coll Cardiol 2006; $47: 362-7$.

2. Zhang Y, Post WS, Dalal D, Blasco-Colmenares E, Tomaselli GF, Guallar E. QT-interval duration and mortality rate: results from the Third National Health and Nutrition Examination Survey. Arch Intern Med 2011;171:172733

3. Beinart R, Zhang Y, Lima JA, et al. The QT interval is associated with incident cardiovascular events: the MESA study. J Am Coll Cardiol 2014;64:2111-9.

4. Schwartz PJ, Wolf S. QT interval prolongation as predictor of sudden death in patients with myocardial infarction. Circulation 1978;57:1074-7. 
5. Peters RW, Byington RP, Barker A, Yusuf S. Prognostic value of prolonged ventricular repolarization following myocardial infarction: the BHAT experience. The BHAT Study Group. J Clin Epidemiol 1990;43:167-72.

6. Bijl M, Verheugt FW. Extreme QT prolongation solely due to reversible myocardial ischemia in single-vessel coronary disease. Am Heart $\mathrm{J}$ 1992;123:524-6.

7. Bonnemeier H, Hartmann F, Wiegand UK, Bode F, Katus HA, Richardt G. Course and prognostic implications of QT interval and QT interval variability after primary coronary angioplasty in acute myocardial infarction. J Am Coll Cardiol 2001;37:44-50.

8. Alici G, Sahin M, Ozkan B, et al. The comparison in reduction of QT dispersion after primary percutaneous coronary intervention according to existence of thrombectomy in ST-segment elevation myocardial infarction. Clin Cardiol 2013;36:276-9.

9. Canavosio FG, Botta M, Bergerone S, Gaita F. Prolonged QT interval in ST-elevation myocardial infarction: predictors and prognostic value in medium-term follow-up. J Cardiovasc Med (Hagerstown) 2016;17:440-5.

10. McLaughlin MG, Stone GW, Aymong E, et al. Prognostic utility of comparative methods for assessment of ST-segment resolution after primary angioplasty for acute myocardial infarction: the Controlled Abciximab and Device Investigation to Lower Late Angioplasty Complications (CADILLAC) trial. J Am Coll Cardiol 2004;44:1215-23.

11. Spitaleri G, Brugaletta S, Scalone G, et al. Role of ST-Segment Resolution in Patients With ST-Segment Elevation Myocardial Infarction Treated With Primary Percutaneous Coronary Intervention (from the 5-Year Outcomes of the EXAMINATION [Evaluation of the Xience-V Stent in Acute Myocardial Infarction] Trial). Am J Cardiol 2018;121:1039-45.
12. Henriques JP, Zijlstra F, van 't Hof AW, et al. Angiographic assessment of reperfusion in acute myocardial infarction by myocardial blush grade. Circulation 2003;107:2115-9.

13. Poli A, Fetiveau R, Vandoni P, et al. Integrated analysis of myocardial blush and ST-segment elevation recovery after successful primary angioplasty: Real-time grading of microvascular reperfusion and prediction of early and late recovery of left ventricular function. Circulation 2002;106:313-8.

14. Ibanez B, James S, Agewall S, et al. 2017 ESC Guidelines for the management of acute myocardial infarction in patients presenting with STsegment elevation: The Task Force for the management of acute myocardial infarction in patients presenting with ST-segment elevation of the European Society of Cardiology (ESC). Eur Heart J 2018;39:119-77.

15. Chesebro JH, Knatterud G, Roberts R, et al. Thrombolysis in Myocardial Infarction (TIMI) Trial, Phase I: A comparison between intravenous tissue plasminogen activator and intravenous streptokinase. Clinical findings through hospital discharge. Circulation. 1987;76:142-54.

16. Lin JF, Hsu SY, Wu S, et al. QT interval Independently Predicts Mortality and Heart Failure in Patients with ST-Elevation Myocardial Infarction. Int J Med Sci 2015;12:968-73.

17. Browne KF, Zipes DP, Heger JJ, Prystowsky EN. Influence of the autonomic nervous system on the Q-T interval in man. Am J Cardiol 1982;50:1099103.

18. Kenigsberg DN, Khanal S, Kowalski M, Krishnan SC. Prolongation of the QTc interval is seen uniformly during early transmural ischemia. J Am Coll Cardiol 2007;49:1299-305.

19. Niwa H, Maeda T, Shimoyama N, et al. [Serial changes in QT interval during acute myocardial infarction: comparison of QT interval changes of reperfused cases and non-reperfused cases]. J Cardiol 1993;23:41-50. 